\title{
Acromegaly: a clinical perspective
}

\author{
Lima Lawrence ${ }^{1 *}$ (D) Kenda Alkwatli ${ }^{1}$, James Bena ${ }^{2}$, Richard Prayson ${ }^{3}$, Varun Kshettry ${ }^{4}$, Pablo Recinos ${ }^{4}$, \\ Betul Hatipoglu' ${ }^{1}$, Kevin M. Pantalone ${ }^{1}$, Robert Weil ${ }^{5}$, Amir H. Hamrahian ${ }^{6}$, Laurence Kennedy ${ }^{1}$ and \\ Divya Yogi-Morren
}

\begin{abstract}
Background: To examine the clinical and hormonal profiles, comorbidities, treatment patterns, surgical pathology and clinical outcomes of patients diagnosed with acromegaly at the Cleveland Clinic over a 15-year period.

Methods: A retrospective chart review of patients with acromegaly who underwent surgical resection between 2003 and 2018.

Results: A total of 136 patients (62 men; mean age 48.1 years) with biochemical evidence of acromegaly were analyzed. Median insulin-like growth factor 1 (IGF-1) level at diagnosis was $769.0 \mathrm{ng} / \mathrm{mL}$ and most patients had a macroadenoma (82.2\%). Immunoreactivity to growth hormone $(\mathrm{GH})$ was noted in 124 adenomas, with co-staining in 89 adenomas. Complete visible tumor resection during initial surgery was achieved in 87 patients (64.0\%). In this cohort, complete response to surgery alone was observed in 61 patients (70.1\%), while 31 out of 65 patients (47.7\%) who received additional post-surgical medications and/or radiation therapy achieved complete response. At most recent follow-up, 92 patients achieved eventual complete response by documented normalization of IGF-1 levels. Higher IGF-1 level at diagnosis $(P=0.024)$ and cavernous sinus invasion $(P=0.028)$ were predictors for failure to respond to surgery.

Conclusion: In this study, the majority of tumors were macroadenoma, plurihormonal, and treated effectively with surgery alone or surgery with adjuvant medical or radiation therapy. More studies are needed to identify additional molecular biomarkers, tumor characteristics and imaging findings to individualize treatment and better predict treatment outcomes.
\end{abstract}

Keywords: Acromegaly, IGF-1, Growth hormone, Pituitary adenoma, Sparsely granulated

\section{Background}

Acromegaly is a rare disease, characterized by excess growth hormone $(\mathrm{GH})$ and insulin-like growth factor 1 (IGF-1) levels usually caused by a somatotroph adenoma of the pituitary gland. The elevated IGF-1 levels causes somatic growth and metabolic effects, with subsequently increased morbidity and mortality, particularly when $\mathrm{GH}$ and IGF-1 levels remain persistently elevated [1]. The

\footnotetext{
* Correspondence: limalawrence@gmail.com

'Department of Endocrinology, Diabetes and Metabolism, Cleveland Clinic, 9500 Euclid Ave. F-20, Cleveland, OH 44195, USA

Full list of author information is available at the end of the article
}

presentation of acromegaly is insidious leading to diagnostic delays.

In patients with suspected acromegaly, the diagnosis is confirmed with biochemical tests including random serum IGF-1, and GH level following a glucose load. A pituitary magnetic resonance imaging (MRI) is performed to assess for evidence of an adenoma, followed by surgical resection as standard of care. About half of patients with macroadenoma do not achieve cure following resection and require repeat surgery, medications or radiation [2]. Medical management of acromegaly may involve use of somatostatin analogs (SSA), dopamine

(c) The Author(s). 2020 Open Access This article is licensed under a Creative Commons Attribution 4.0 International License, which permits use, sharing, adaptation, distribution and reproduction in any medium or format, as long as you give appropriate credit to the original author(s) and the source, provide a link to the Creative Commons licence, and indicate if changes were made. The images or other third party material in this article are included in the article's Creative Commons licence, unless indicated otherwise in a credit line to the material. If material is not included in the article's Creative Commons licence and your intended use is not permitted by statutory regulation or exceeds the permitted use, you will need to obtain permission directly from the copyright holder. To view a copy of this licence, visit http://creativecommons.org/licenses/by/4.0/ The Creative Commons Public Domain Dedication waiver (http://creativecommons.org/publicdomain/zero/1.0/) applies to the data made available in this article, unless otherwise stated in a credit line to the data. 
agonists (DA), growth hormone receptor antagonist pegvisomant (PEG) or combination therapy.

The aim of this study is to examine the clinical and hormonal profiles, comorbidities, complications, surgical pathology, treatment patterns, clinical outcomes, and mortality of patients diagnosed with acromegaly and surgically treated at the Cleveland Clinic over a 15-year period from 2003 to 2018.

\section{Methods}

\section{Study design and patient selection}

A retrospective chart review of all patients who were diagnosed with acromegaly in our institution over a 15 -year period was undertaken. After approval from the Cleveland Clinic Institutional Review Board, we reviewed 1122 charts from a pituitary pathology database with surgically resected pituitary adenomas from 2003 to 2018. We selected patients from the pituitary pathology database who had a pituitary adenoma with evidence of GH staining on pathology, as well as patients who had clinical and biochemical evidence of acromegaly despite negative $\mathrm{GH}$ staining on pathology. Those who did not meet the above criteria were excluded. A total of 136 (12.1\%) patients were identified for inclusion in the study.

We collected demographic information, clinical history including signs and symptoms at presentation, comorbidities, time since onset of symptoms and date of diagnosis. Additional data included biochemical lab results, tumor measurements, tumor characteristics on MRI, pathology information including pituitary hormone costaining and granulation pattern, pre-operative medical treatment, completeness of initial tumor resection, medications initiated after surgery, and need for repeat surgery or irradiation. We also evaluated response to surgery, medication and radiation, along with patientreported improvement in symptoms after treatment, and objective documentation of improvement in comorbidities. Complete initial tumor resection was defined as surgical removal of all visible tumor. Complete response to surgery was defined as radiographic absence of residual tumor and normalization of IGF-1 and GH levels (defined as random $\mathrm{GH}<1.0 \mathrm{ng} / \mathrm{mL}$ ) on last follow-up testing, while complete response to medications and irradiation therapy was defined as normalization of IGF1 and GH levels on last follow-up testing regardless of residual tumor.

\section{Biochemical data}

Laboratory data was mostly obtained from the Cleveland Clinic Main Laboratory, while some values were obtained from outside hospital records. Pre-operative data were obtained for serum GH $(\mathrm{ng} / \mathrm{mL})$, IGF-1 $(\mathrm{ng} / \mathrm{mL})$ and 2-h post oral glucose tolerance test (OGTT) GH (< $1.0 \mathrm{ng} / \mathrm{mL}$ ). GH measurement was performed using quantitative chemiluminescence immunoassay (ARUP). Biochemical evidence of acromegaly was established with IGF-1 level above age-appropriate normal value, and nadir serum GH $>1 \mathrm{ng} / \mathrm{mL}$ post OGTT based on Endocrine Society and American Association of Clinical Endocrinologists (AACE) guidelines on acromegaly diagnosis. Although current GH assays have improved sensitivity, many assays do not have sufficient accuracy at $\mathrm{GH}$ levels $<1 \mathrm{ng} / \mathrm{mL}$ and there is poor reproducibility between laboratories and assays. As we reviewed laboratory data extending over 15 years from within and outside of our institution, we decided against using lower 2h post-OGTT GH cutoffs in establishing the biochemical diagnosis of acromegaly.

\section{Imaging}

Computed tomography (CT) or MRI reports were reviewed before and after surgical resection of pituitary adenoma. Based on the radiology report available in our electronic health record system or exported from outside institution, we obtained data on tumor diameter, tumor invasion and extension into cavernous sinus (CS) or optic chiasm (OC), and completeness of tumor resection post-surgery.

\section{Surgical procedures}

All pituitary surgeries were performed by three dedicated skull-base neurosurgeons with or without involvement of an otolaryngologist. Surgical approaches included transsphenoidal approach with microscopy in earlier cases and endoscopic transnasal transphenoidal approach subsequently. In few select patients, repeat craniotomy $(n=3)$ was required. In all patients, the goal of surgery was gross total resection of all visible tumor, or maximal debulking in large and giant extended adenomas.

\section{Pathology evaluation}

Surgical pathology was interpreted by neuropathologists at the Cleveland Clinic. Immunohistochemical staining with antibodies to pituitary hormones was routinely performed on pituitary adenomas. Antibodies evaluated included the following: anti-GH (1:2500 dilution; DAKO), anti-TSH (prediluted; Cell Marque, Rocklin, CA), antiACTH (1:2500 dilution; Thermo Scientific, Waltham, MA), anti-FSH (1:200 dilution; DAKO, Carpinteria, CA), anti-LH (1:320 dilution; DAKO) and anti-PRL (1:800 dilution; DAKO) with positive and negative control evaluated with each antibody stain in each case. Cytokeratin CAM5.2 staining (1:10 dilution; BD, San Jose, CA) was routinely used after 2015 to classify adenomas based on distribution pattern of keratin filaments into densely granulated (DG) or sparsely granulated (SG) adenomas. DG adenomas display a perinuclear or non-dot pattern with cytokeratin immunostaining under light microscopy; while 
SG adenomas show dot-like cytokeratin pattern with numerous CAM5.2 immunohistochemistry-positive fibrous bodies [3]. During data collection, based on pathology report, we classified GH staining as rare or diffuse, and if present, pituitary hormone co-staining evaluation for thyroid-stimulating hormone (TSH), prolactin (PRL), adrenocorticotropic hormone (ACTH) and folliclestimulating hormone (FSH).

\section{Statistical analysis}

For analysis of demographics, labs, tumor properties, evaluation of treatment and outcomes, categorical factors were summarized using frequencies and percentages, while continuous measures were evaluated for normality graphically and using the Shapiro-Wilk test. Normally distributed measures were summarized using means and standard deviations, while non-normal measures were summarized with medians and quartiles.

For univariable analyses, categorical factors were summarized using frequencies and percentages, and comparisons used Pearson's chi-square or Fisher's Exact tests, while continuous measures were evaluated for normality graphically and using the Shapiro-Wilk test. Normally distributed measures were summarized using means and standard deviations, and comparisons used ANOVA tests, while non-normal measures were summarized with medians, quartiles or ranges, and comparisons used Kruskal-Wallis tests. Odds ratios (OR) were calculated for non-zero responses.

A stepwise regression was performed for multivariable analyses for incomplete resection and persistent elevation in IGF-1 levels post-resection, given the low number of events for each outcome relative to the number of potential risk factors. Factors with a $P$-value less than 0.2 and at least $90 \%$ non-missing data were considered for the models. Variables were entered into the model if they had a $P$-value less than 0.10 and remained in the model if their P-value was under 0.05 . After finalizing the stepwise methods, the models were refit with just the chosen variables to remove the impact of other variables on observations. OR and 95\% confidence intervals were presented for each model. As a sensitivity analysis, penalized logistic regression using Firth's methods was used to evaluate whether the models might be overfit.

A $P$-value of $<0.05$ was predetermined as statistically significant. All models were fit and analyses were performed using SAS software (version 9.4; Cary, NC).

\section{Results}

\section{Patients}

Among the 1122 pituitary adenomas, 136 (12.1\%) demonstrated biochemical evidence of acromegaly and 124 stained positive for GH on histology. Sixty-two men (45.6\%) and 74 women (54.4\%) were diagnosed at mean age of $48.1 \pm 13.8$ years. A majority of patients reported onset of symptoms at mean age $40.5 \pm 13.8$ years, with average diagnostic delay of 7.6 years. Available mortality data revealed $94.1 \%$ of patients were alive at the time of data collection based on our institution's electronic health records.

Table 1 details the demographics and presenting symptoms. Musculoskeletal complaints (acral or facial enlargement, dental problems, osteoarthritis, carpal tunnel syndrome) were reported by $89.6 \%$ of patients, compressive symptoms (headache, visual changes or cranial nerve deficits) by $65.6 \%$ of patients, and hypogonadism (amenorrhea, sexual dysfunction, gynecomastia, galactorrhea) by $45.2 \%$ of patients. Miscellaneous concerns including hyperhidrosis, acne, hirsutism and dizziness were reported by $58.3 \%$ of patients, while $40.6 \%$ complained of psychological symptoms including mood changes and depression. Only a minority of patients (12.1\%) were incidentally discovered to have a sellar mass on imaging as the presenting feature.

Patients presented with comorbidities including hypertension (56.7\%), obstructive sleep apnea (47.5\%) and diabetes mellitus (44.4\%). Cardiac dysfunction including coronary artery disease, congestive heart failure or cardiomyopathy was observed in $16.8 \%$ of patients, while $12.9 \%$ had a history of, or were subsequently diagnosed with, malignancy (Table 1). Multiple endocrine neoplasia type-1 was confirmed with genetic testing in one patient.

Table 2 details the biochemical findings and tumor characteristics on presentation. Median (minimum to maximum range) IGF-1 level at diagnosis was $769.0 \mathrm{ng} /$ $\mathrm{mL}$ ( 235 to $1845 \mathrm{ng} / \mathrm{mL}$ ), with median baseline GH level of $8.2 \mathrm{ng} / \mathrm{mL}$ ( 0.17 to $808.12 \mathrm{ng} / \mathrm{mL}$ ), and median nadir 2-h post-OGTT GH level of $4.4 \mathrm{ng} / \mathrm{mL}$ ( 0.2 to $330.0 \mathrm{ng}$ / $\mathrm{mL})$. Most patients were discovered to have a macroadenoma (82.2\%), with median tumor dimensions of 16.0 (antero-posterior) $\times 16.0$ (transverse) $\times 14.0$ (craniocaudal) $\mathrm{mm}$. CS invasion (44.7\%) and OC compression (32.6\%) were noted on MRI. GH staining was positive in 124 adenomas (91.2\%). Out of 65 adenomas with reported staining pattern, 36 demonstrated diffuse staining for $\mathrm{GH}(55.4 \%)$, while 29 demonstrated rare GH staining pattern (44.6\%). Pituitary hormone co-staining was observed in 89 adenomas (65.4\%), including 57 adenomas staining for both TSH and prolactin (PRL), 12 staining for ACTH, and 4 staining for FSH. Cytokeratin CAM5.2 staining performed in 23 patients revealed 17 DG and 6 SG adenomas.

\section{Treatment and outcomes}

Eighty-four patients (62.7\%) received pre-surgical medical treatment as follows: octreotide $(80.9 \%)$ or lanreotide (7.1\%) alone, followed by combination therapy with octreotide and cabergoline (4.8\%), while 2 patients 
Table 1 Patient Demographics, Symptoms and Comorbidities at Presentation

\begin{tabular}{|c|c|c|}
\hline \multirow[t]{2}{*}{ Factor } & \multicolumn{2}{|c|}{$\begin{array}{l}\text { Total } \\
(\boldsymbol{N}=136)\end{array}$} \\
\hline & $n$ & $\mathrm{n}(\%)$ or Mean $\pm \mathrm{SD}$ \\
\hline Sex & 136 & \\
\hline - Male & & $62(45.6)$ \\
\hline - Female & & $74(54.4)$ \\
\hline Age at presenting symptoms & 81 & $40.5 \pm 14.2$ \\
\hline Age at diagnosis & 136 & $48.1 \pm 13.8$ \\
\hline Mortality status & 136 & \\
\hline - Deceased & & $8(5.9)$ \\
\hline - Alive & & 128(94.1) \\
\hline Hypogonadism & 126 & $57(45.2)$ \\
\hline Compression symptoms & 131 & $86(65.6)$ \\
\hline Musculoskeletal symptoms & 135 & $121(89.6)$ \\
\hline Psychological symptoms & 128 & $52(40.6)$ \\
\hline${ }^{a}$ Miscellaneous symptoms & 132 & $77(58.3)$ \\
\hline Incidental CT/MRI finding & 132 & $16(12.1)$ \\
\hline Diabetes mellitus & 133 & $59(44.4)$ \\
\hline Hypertension & 134 & $76(56.7)$ \\
\hline Obstructive sleep apnea & 122 & $58(47.5)$ \\
\hline Coronary artery disease / congestive heart failure / cardiomyopathy & 125 & 21(16.8) \\
\hline Malignancy & 132 & 17(12.9) \\
\hline Genetic confirmation of MEN1 syndrome & 136 & $1(0.74)$ \\
\hline
\end{tabular}

${ }^{a}$ Miscellaneous symptoms including hyperhidrosis, acne, hirsutism and dizziness

received PEG (Table 3). All 136 patients underwent pituitary tumor resection; four patients had initial craniotomy (2.9\%), while 132 patients underwent transsphenoidal resection (97.1\%). Complete visible tumor resection during initial surgery was achieved in 87 patients (64.0\%). Subsequently, 19 patients required repeat surgery (of which three were open craniotomy), and 27 patients underwent irradiation.

Medical therapy post-surgery was initiated in 65 patients (47.8\%), with 32 patients receiving SSA alone (49.2\%), 3 patients receiving DA alone (4.6\%), while 11 received combination therapy with SSA, DA and PEG (16.9\%), and 1 patient received SSA, DA, PEG and pasireotide in step-wise escalation of therapy (Table 3). Complete visible tumor resection during initial surgery was achieved in 87 patients (64.0\%). In this cohort, complete response to surgery alone was observed in 61 patients $(70.1 \%)$, while 31 patients out of $65(47.7 \%)$ who received subsequent post-surgical medications and/or radiation therapy achieved complete response. At most recent follow-up, 92 out of the total cohort of 136 patients (67.6\%) achieved eventual complete response by documented normalization of IGF-1 levels. Overall, a significant majority of patients reported improvement in symptoms (91.5\%) and had objective improvement in comorbidities $(69.0 \%)$ after acromegaly treatment. The development of postsurgical hypopituitarism was noted in 22 out of 133 patients (16.5\%). All patients who developed hypopituitarism had a history of irradiation, and 16 out of 22 patients (72.7\%) with hypopituitarism had undergone repeat surgery.

\section{Univariable analyses with odds ratios}

Table 4 details the outcomes of univariable analyses based on completeness of initial surgical tumor resection. Patients with complete initial resection of visible tumor had lower GH levels at diagnosis $(P=0.001)$ and smaller tumors in all dimensions (all $P<0.001$ ). For each $1 \mathrm{ng} / \mathrm{mL}$ unit increase in $\mathrm{GH}$, the odds of complete tumor resection decreased by $2 \%$ (OR (95\% CI): 0.98 $(0.97,1.00), P=0.001)$. Those with complete initial resection were also more likely to have GH staining $(P=$ $0.022)$ and pituitary hormone co-staining $(P=0.026)$. They were less likely to have CS invasion and OC compression (both $P<0.001$ ) or develop hypopituitarism $(\mathrm{P}<0.001)$. The odds of a patient with hypopituitarism having had complete initial resection were $92 \%$ less than a patient without hypopituitarism (OR (95\% CI): 0.08 $(0.03,0.27), \mathrm{P}<0.001)$. 
Table 2 Labs and Tumor Properties on Imaging and Histopathology

\begin{tabular}{|c|c|c|}
\hline \multirow[t]{2}{*}{ Factor } & \multicolumn{2}{|c|}{$\begin{array}{l}\text { Total } \\
(\boldsymbol{N}=136)\end{array}$} \\
\hline & $\mathrm{n}$ & $\mathrm{n}(\%)$ or Median [P25, P75] \\
\hline Random GH level at Dx & 125 & $8.2[3.2,34.0]$ \\
\hline IGF-1 level at Dx & 134 & $769.0[567.0,956.0]$ \\
\hline 2-h nadir post OGTT GH at Dx & 44 & $4.4[1.6,16.9]$ \\
\hline Most recent random GH level & 128 & $0.61[0.31,1.8]$ \\
\hline Most recent IGF-1 level & 134 & $205.5[155.0,281.0]$ \\
\hline Most recent 2-h nadir post OGTT GH & 32 & $0.32[0.19,0.90]$ \\
\hline Months between GH measures & 116 & $21.5[7.9,65.3]$ \\
\hline Months between IGF-1 measures & 131 & $34.7[12.8,87.9]$ \\
\hline Tumor size & 135 & \\
\hline - Macroadenoma & & $111(82.2)$ \\
\hline - Microadenoma & & $24(17.8)$ \\
\hline Tumor length/antero-posterior & 126 & $16.0[11.0,22.0]$ \\
\hline Tumor width/transverse & 116 & $16.0[10.5,20.0]$ \\
\hline Tumor height/craniocaudal & 105 & $14.0[10.0,20.0]$ \\
\hline GH staining & 136 & 124(91.2) \\
\hline GH staining type & 65 & \\
\hline - Rare & & 29(44.6) \\
\hline - Diffuse & & $36(55.4)$ \\
\hline Pituitary hormone co-staining & 136 & $89(65.4)$ \\
\hline$\cdot$ TSH & 136 & $57(41.9)$ \\
\hline - Prolactin & 136 & $57(41.9)$ \\
\hline • ACTH & 136 & $12(8.8)$ \\
\hline • FSH & 136 & $4(2.9)$ \\
\hline Granulation pattern & 23 & \\
\hline - Densely & & 17(73.9) \\
\hline - Sparsely & & $6(26.1)$ \\
\hline Cavernous sinus invasion & 132 & $59(44.7)$ \\
\hline Optic chiasm compression & 132 & 43(32.6) \\
\hline
\end{tabular}

Outcomes of univariable analyses based on response to surgery in those with complete initial resection were performed. Complete response to surgery was defined as normalization of IGF-1 levels without evidence of residual tumor at the time of last follow-up. Patients who responded to surgery had lower GH $(P=0.027)$ and lower IGF-1 levels $(P=0.001)$ at diagnosis, along with lower GH level $(P=0.031)$ and 2 -h nadir post-OGTT GH level $(P=0.014)$ at most recent follow-up. Lastly, patients who responded to surgery were less likely to have CS invasion $(P=0.015)$. Outcomes of univariable analyses based on response to medication in those with complete initial resection were performed. Response to medication was defined as normalization of IGF-1 levels at last follow-up. Patients who responded to medication
Table 3 Treatment and Patient Outcomes

\begin{tabular}{|c|c|c|}
\hline \multirow[t]{2}{*}{ Factor } & \multicolumn{2}{|c|}{$\begin{array}{l}\text { Total } \\
(\boldsymbol{N}=136)\end{array}$} \\
\hline & $n$ & n (\%) \\
\hline Preoperative medical treatment & 134 & $84(62.7)$ \\
\hline - Octreotide & 84 & 68(80.9) \\
\hline - Lanreotide & 84 & $6(7.1)$ \\
\hline - Bromocriptine & 84 & $1(1.2)$ \\
\hline - Cabergoline & 84 & $2(2.4)$ \\
\hline - Pegvisomant & 84 & 0 \\
\hline - Octreotide + Bromocriptine & 84 & $1(1.2)$ \\
\hline - Octreotide + Cabergoline & 84 & $4(4.8)$ \\
\hline - Octreotide + Pegvisomant & 84 & $2(2.4)$ \\
\hline Transsphenoidal surgery & 136 & 135(99.3) \\
\hline Craniotomy required & 132 & $7(5.3)$ \\
\hline Initial complete resection & 135 & $87(64.4)$ \\
\hline Repeat surgery & 136 & 19(14.0) \\
\hline Irradiation & 135 & $27(20.0)$ \\
\hline Medication initiated after surgery & 136 & $65(47.8)$ \\
\hline - Somatostatin analog (SSA) & 65 & $32(49.2)$ \\
\hline - Dopamine agonists (DA) & 65 & $3(4.6)$ \\
\hline - Pasireotide & 65 & 0 \\
\hline • Pegvisomant (PEG) & 65 & $1(1.5)$ \\
\hline - Combination SSA + DA & 65 & $8(12.3)$ \\
\hline - Combination SSA + PEG & 65 & $7(10.8)$ \\
\hline - Combination DA + PEG & 65 & $2(3.1)$ \\
\hline - Sequential SSA + DA + PEG & 65 & $11(16.9)$ \\
\hline - Sequential SSA + DA + PEG + Pasireotide & 65 & $1(1.5)$ \\
\hline Response to surgery & 87 & $61(70.1)$ \\
\hline Response to medication and/or radiation: & 65 & $31(47.7)$ \\
\hline Hypopituitarism & 133 & $22(16.5)$ \\
\hline Improvement of symptoms after treatment & 118 & 108(91.5) \\
\hline Improvement of comorbidities after treatment & 87 & $60(69.0)$ \\
\hline
\end{tabular}

had lower levels of GH $(P=0.003)$ and IGF-1 $(P=0.046)$ at the time of their most recent follow-up.

Outcomes of univariable analyses of those who underwent repeat surgery were performed. Patients with repeat surgery were younger $(P=0.026)$, had higher $\mathrm{GH}$ levels at diagnosis $(P=0.011)$, larger tumors (anteroposterior $P<0.001$, transverse $P=0.002$, and craniocaudal $\mathrm{P}=0.002)$, with $\mathrm{CS}$ invasion $(P=0.044)$ and $\mathrm{OC}$ compression $(P=0.011)$. Patients who underwent repeat surgery were less likely to have pituitary hormone costaining with TSH $(P=0.047)$. Repeat surgery increased the risk of hypopituitarism $(P<0.001)$, with hypopituitarism present in 16 out of 19 patients (84.2\%).

Outcomes of univariable analyses based on response to medication in those who had incomplete initial tumor 
Table 4 Labs and Tumor Properties of Patients with Complete Initial Resection

\begin{tabular}{|c|c|c|c|c|c|c|}
\hline \multirow[t]{2}{*}{ Factor } & \multicolumn{2}{|c|}{$\begin{array}{l}\text { Incomplete } \\
(\boldsymbol{N}=49)\end{array}$} & \multicolumn{2}{|c|}{$\begin{array}{l}\text { Complete } \\
(\boldsymbol{N}=86)\end{array}$} & \multirow[b]{2}{*}{$\boldsymbol{p}$-value } & \multirow[b]{2}{*}{ Odds Ratios $(95 \% \mathrm{Cl})$} \\
\hline & $\mathrm{n}$ & $\begin{array}{l}\mathrm{n}(\%) \text { or } \\
\text { Median [P25, P75] }\end{array}$ & $\mathrm{n}$ & $\begin{array}{l}\mathrm{n}(\%) \text { or } \\
\text { Median [P25, P75] }\end{array}$ & & \\
\hline Random GH level at Dx & 46 & $25.4[4.5,62.9]$ & 78 & $6.3[2.6,21.1]$ & $0.001^{b}$ & $0.98(0.97,1.00)$ \\
\hline IGF-1 level at Dx & 49 & $784.0[675.0,1014.0]$ & 84 & $752.0[528.5944 .0]$ & $0.30^{\mathrm{b}}$ & $1.00(1.00,1.00)$ \\
\hline 2-h nadir post OGTT GH at Dx & 12 & $8.2[1.8,29.7]$ & 31 & $4.3[1.7,12.8]$ & $0.59^{b}$ & $1.00(0.99,1.01)$ \\
\hline Most recent random GH level & 45 & $1.4[0.32,2.2]$ & 82 & $0.59[0.30,1.6]$ & $0.11^{\mathrm{b}}$ & $0.99(0.96,1.02)$ \\
\hline Most recent IGF-1 level & 49 & $217.0[153.0,377.0]$ & 84 & $205.5[161.0,272.5]$ & $0.50^{b}$ & $1.00(1.00,1.00)$ \\
\hline Most recent 2-h nadir post OGTT GH & 9 & $0.51[0.21,1.3]$ & 23 & $0.30[0.16,0.80]$ & $0.35^{\mathrm{b}}$ & $0.74(0.26,2.1)$ \\
\hline Tumor size & 49 & & 85 & & $0.077^{c}$ & \\
\hline - Macroadenoma & & $44(89.8)$ & & $66(77.6)$ & & reference \\
\hline • Microadenoma & & $5(10.2)$ & & $19(22.4)$ & & $2.5(0.88,7.3)$ \\
\hline Tumor length/antero-posterior & 43 & 22.0[14.0,29.0] & 82 & $14.5[10.0,18.0]$ & $<0.001^{b}$ & $0.92(0.88,0.96)$ \\
\hline Tumor width/transverse & 42 & $20.5[15.0,25.0]$ & 74 & $14.5[10.0,18.0]$ & $<0.001^{b}$ & $0.89(0.84,0.94)$ \\
\hline Tumor height/craniocaudal & 39 & $20.0[15.0,25.0]$ & 66 & $13.0[9.0,16.0]$ & $<0.001^{b}$ & $0.86(0.80,0.92)$ \\
\hline GH staining & 49 & $41(83.7)$ & 86 & $82(95.3)$ & $0.022^{c}$ & $4.0(1.1,14.1)$ \\
\hline GH staining type & 25 & & 39 & & $0.11^{c}$ & \\
\hline - Rare & & $14(56.0)$ & & 14(35.9) & & reference \\
\hline - Diffuse & & $11(44.0)$ & & $25(64.1)$ & & $2.3(0.81,6.3)$ \\
\hline Pituitary hormone co-staining & 49 & $26(53.1)$ & 86 & $62(72.1)$ & $0.026^{c}$ & $2.3(1.10,4.8)$ \\
\hline$\cdot$ TSH & 49 & 15(30.6) & 86 & $41(47.7)$ & $0.053^{c}$ & $2.1(0.98,4.3)$ \\
\hline - Prolactin & 49 & $16(32.7)$ & 86 & $40(46.5)$ & $0.12^{c}$ & $1.8(0.86,3.7)$ \\
\hline$\cdot \mathrm{ACTH}$ & 49 & $2(4.1)$ & 86 & 10(11.6) & $0.14^{c}$ & $3.1(0.65,14.7)$ \\
\hline • FSH & 49 & $0(0.0)$ & 86 & $4(4.7)$ & $0.30^{\mathrm{d}}$ & NA \\
\hline Granulation pattern & 8 & & 15 & & $0.13^{\mathrm{d}}$ & \\
\hline - Densely & & $4(50.0)$ & & 13(86.7) & & reference \\
\hline - Sparsely & & $4(50.0)$ & & $2(13.3)$ & & $0.15(0.02,1.2)$ \\
\hline Cavernous sinus invasion & 46 & $31(67.4)$ & 85 & $28(32.9)$ & $<0.001^{c}$ & $0.24(0.11,0.51)$ \\
\hline Optic chiasm compression & 47 & 25(53.2) & 84 & $17(20.2)$ & $<0.001^{c}$ & $0.22(0.10,0.49)$ \\
\hline
\end{tabular}

p-values: $\mathrm{b}=$ Kruskal-Wallis test, $\mathrm{c}=$ Pearson's chi-square test, $\mathrm{d}=$ Fisher's Exact test

resection were examined. Patients who responded to subsequent medical therapy were more likely to be female $(P=0.008)$, older at onset of symptoms and age at diagnosis (both $P<0.001$ ), and less likely to report compressive symptoms on presentation $(P=0.022)$. These patients also had lower GH level $(\mathrm{P}<0.001)$ at most recent follow-up. Outcomes of univariable analyses based on improvement in symptoms and comorbidities were performed. Patients who reported improvement in symptoms were more likely to be older at onset of symptoms $(P=0.036)$ and age at diagnosis $(P=0.023)$, and less likely to have hypopituitarism $(P=0.046)$. Patients who had objective improvement in comorbidities were more likely to be female and older at diagnosis (both $P=0.0 .023)$. They had smaller tumor transverse dimension on presentation $(P=0.008)$ and less likely to have CS invasion $(\mathrm{P}=0.008)$ and $\mathrm{OC}$ compression $(P=0.029)$.
These patients also were more likely to have tumors stain positive for GH $(P=0.015)$ and co-stain with TSH $(P=0.036)$. Patients with improvement in comorbidities were less likely to have hypopituitarism $(P<0.001)$.

Subgroup analyses in Table 5 reveals that patients with SG adenomas were more likely to require repeat surgery than those with DG adenomas $(P=0.040)$.

\section{Multivariable analyses}

Multivariable models for incomplete surgical resection and failure to respond to surgery among those with complete resection were analyzed. Predictors for failing to achieve complete initial tumor resection are CS invasion $(P=0.048)$ and $\mathrm{OC}$ compression $(P=0.023)$. Patients with CS invasion have 2.4 times higher odds of failure to achieve complete resection compared to patients without CS invasion, after adjustment for other 
Table 5 Outcomes of Patients with Densely and Sparsely Granulated Adenomas

\begin{tabular}{|c|c|c|c|c|c|c|}
\hline \multirow[t]{2}{*}{ Factor } & \multicolumn{2}{|c|}{$\begin{array}{l}\text { Densely } \\
(\boldsymbol{N}=17)\end{array}$} & \multicolumn{2}{|c|}{$\begin{array}{l}\text { Sparsely } \\
(\boldsymbol{N}=6)\end{array}$} & \multirow[b]{2}{*}{$\boldsymbol{p}$-value } & \multirow[b]{2}{*}{$\begin{array}{l}\text { Odds Ratios } \\
(95 \% \mathrm{Cl})\end{array}$} \\
\hline & $\mathrm{n}$ & n (\%) & $\mathrm{n}$ & n (\%) & & \\
\hline Initial complete resection & 17 & 13(76.5) & 6 & 2(33.3) & $0.13^{\mathrm{d}}$ & $0.15(0.02,1.2)$ \\
\hline Repeat surgery & 17 & $1(5.9)$ & 6 & $3(50.0)$ & $0.040^{d}$ & $16.0(1.2,210.6)$ \\
\hline Response to surgery & 13 & $9(69.2)$ & 2 & $0(0.0)$ & $0.14^{d}$ & NA \\
\hline Response to medication and/or radiation: & 5 & $2(40.0)$ & 5 & $1(20.0)$ & $0.99^{d}$ & $0.38(0.02,6.3)$ \\
\hline Improvement of symptoms after treatment & 16 & 16(100.0) & 4 & $4(100.0)$ & N/A & $\mathrm{N} / \mathrm{A}$ \\
\hline Improvement of comorbidities after treatment & 10 & 10(100.0) & 3 & $3(100.0)$ & N/A & N/A \\
\hline
\end{tabular}

$p$-values: $\mathrm{d}=$ Fisher's Exact test

terms in this model. Additionally, subsequent development of hypopituitarism is statistically correlated $(P<$ $0.001)$ with incomplete initial tumor resection. Predictors of failure to respond to surgery after complete initial visible tumor resection are higher IGF-1 level at diagnosis $(P=0.024)$ and $C S$ invasion $(P=0.028)$. Significantly, for each $10 \mathrm{ng} / \mathrm{mL}$ unit increase in IGF-1 at diagnosis, the odds of failure to respond to surgery increased by $2 \%$, after adjusting for other factors. The development of hypopituitarism $(P=0.047)$ is correlated with failure to respond to surgery.

\section{Discussion}

Our study demonstrates that patient demographics, initial biochemical findings, tumor characteristics and surgical pathology determine clinical outcomes in patients with acromegaly. The most prevalent presenting complaints and comorbidities in our study are consistent with current literature [4]. The overall low mortality rate $(5.9 \%)$ mirrors the decline in acromegaly mortality rate over the past 30 years [5]. We observed a diagnostic delay of approximately 7 years, similar to findings reported in international acromegaly registries [6-8] and longitudinal studies [9]. Macroadenomas predominated in our cohort $(82.2 \%)$ with associated CS invasion or OC compression, consistent with existing literature $[4,5,9,10]$.

The Endocrine Society and AACE guidelines on acromegaly management recommend pretreatment in a subset of acromegaly patients to improve postsurgical outcomes - for example, those with severe pharyngeal thickness, sleep apnea or high-output heart failure [11, 12]. In our study, a majority of patients received pre-operative medical therapy (62.7\%) prior to surgical resection. Patients in our study who had complete visible tumor resection during initial surgery had lower levels of GH at diagnosis, smaller tumors, lacked CS invasion or OC compression, and were less likely to develop hypopituitarism. Factors associated with increased likelihood of surgical failure in our study is similar to findings in a large Korean study which noted that surgical failure was associated with younger age, larger tumor size and CS invasion [13]. European registries note a prevalence of hypopituitarism ranging from 26 to $34 \%$ in patients with acromegaly, which although higher than that in our study $(16.5 \%)$, is an improvement from the past due to advances in surgical techniques, newer medications, and decreased use of radiotherapy [14-17]. A systematic review examining predictors of quality of life in acromegaly patients reported that hypopituitarism was not significantly correlated with quality of life [18]. However, our study found that patients with self-reported improvement in symptoms were significantly less likely to have hypopituitarism.

Cuevas-Ramos et al. proposed a structural and functional acromegaly classification partially based on granulation pattern: DG adenomas more commonly seen in older patients with longest follow-up and favorable outcomes and SG adenomas characterized by aggressive macroadenomas with adverse therapeutic outcomes despite multistep therapy [19]. In our study, we found that patients who required repeat surgery were significantly more likely to have SG adenomas $(P=0.040)$. Multiple studies have endorsed that patients with SG adenomas are younger, more likely to be female, with higher baseline IGF-1 and GH levels, have significantly larger tumors with aggressive features, and less likely to achieve biochemical normalization in response to surgery or SSA therapy, but exhibit therapeutic response to GH receptor antagonist therapy with PEG [20-26]. Therefore, $\mathrm{GH}$ adenoma granulation pattern may help guide acromegaly therapy. However, the current guidelines on the medical management of acromegaly continue to recommend first-generation long-acting SSA as first-line therapy in those with persistent disease after surgery, cabergoline as adjunct therapy, or transition to pasireotide or PEG based on glycemic status [11, 12, 27]. Therefore, updated guidelines that take into account the genetic, biochemical and histopathological variations in acromegaly, along with patient characteristics and imaging features that determine responsiveness to treatment are necessary. A recently published systematic 
review and consensus treatment guideline takes into account three factors in formulating a personalized approach to medical therapy of acromegaly: granulation pattern, somatostatin receptor 2 (SSTR2) staining and T2 intensity on MRI [28]. Based on their proposed decision tree, patients with DG tumors with high SSTR2 expressivity and T2 hypo-intense or iso-intense tumors on MRI would benefit from SSA therapy [28]. On the other hand, patients with SG tumors with low SSTR2 expressivity and hyperintense T2-weighted MRI signal would benefit from DA therapy when there is only modest elevation of IGF-1, or PEG in the setting of significantly elevated IGF-1 levels, uncontrolled diabetes, and when tumor location and size is not a concern [28]. An accompanying commentary notes that this individualized approach to acromegaly is an improvement over the current "trial-and-assess" and "trial-and-failure" methods, avoiding prolonged futile and expensive treatments, and allowing earlier initiation of newer, more effective medications [29]. This personalized approach in acromegaly management is particularly relevant to those whose disease remains challenging to treat: patients with delayed diagnosis, ineligible for surgery, with persistent or recurrent disease after surgery [30].

Given the retrospective nature of this research, our analyses were primarily exploratory and carry with them potential limitations. From a statistical standpoint, the number of tests performed and use of stepwise modeling procedures both can inflate type I error rates and lead to spurious findings. However, we chose to include all analyses with raw, rather than adjusted, $p$-values so that the reader can use a preferred threshold for significance. Given these concerns, we recognize that these findings should be confirmed in other cohorts. Our report includes patients selected from a pituitary pathology database, which may be an underrepresentation of the total population of acromegaly patients treated at our institution who may have had surgical resection elsewhere, or were poor surgical candidates and were therefore managed medically or with irradiation therapy only. Although our study collected multiple variables, we were unable to quantify all factors which could have impacted choice of therapy and results. We also lack an objective method to assess patient compliance with therapy beyond physician documentation. Furthermore, we have inadequate numbers of DG and SG adenomas necessary to power statistically significant conclusions. However, strengths of our study include presentation of data from a robust number of patients with acromegaly treated in a tertiary care center for 15 years. Our enterprise-wide electronic health records provided a substantial amount of clinical data for us to collect and report.

\section{Conclusion}

In this study, we report our experience with acromegaly in a large tertiary care center. Complete response to surgery alone was observed in 61 patients $(70.1 \%)$, while 31 out of 65 patients (47.7\%) achieved complete response with subsequent post-surgical medications and irradiation therapy. Predictors of failure to respond to surgery after complete initial resection were higher IGF1 level at diagnosis and CS invasion. Patients with SG adenomas were more likely to require repeat surgery $(P=0.040)$; confirming the need for consideration of granulation pattern in individualizing the treatment of acromegaly.

\section{Abbreviations \\ ACTH: Adrenocorticotropic hormone; CS: Cavernous sinus; DA: Dopamine agonist; DG: Densely granulated; FSH: Follicle-stimulating hormone: GH: Growth hormone; IGF-1: Insulin-like growth factor 1; LH: Luteinizing hormone; MRI: Magnetic resonance imaging; OC: Optic chiasm; OGTT: Oral glucose tolerance test; OR: Odds ratio; PEG: Pegvisomant; PRL: Prolactin; SG: Sparsely granulated; SSA: Somatostatin analog; SSTR2: Somatostatin receptor 2; TSH: Thyroid-stimulating hormone}

\section{Acknowledgements}

Not applicable.

\section{Authors' contributions}

Each author has participated in the study design, data collection, statistical analysis and/or manuscript writing and provided final approval for submission and publication.

\section{Funding}

Not applicable.

Availability of data and materials

All data generated or analyzed during this study are included in this published article.

Ethics approval and consent to participate

This study was reviewed and approved by the Cleveland Clinic Institutional Review Board

\section{Consent for publication}

Not applicable.

\section{Competing interests}

The authors declare that they have no competing interests.

\section{Author details}

'Department of Endocrinology, Diabetes and Metabolism, Cleveland Clinic, 9500 Euclid Ave. F-20, Cleveland, OH 44195, USA. ²Department of Quantitative Health Sciences, Cleveland Clinic, Cleveland, OH, USA.

${ }^{3}$ Department of Anatomic Pathology, Cleveland Clinic, Cleveland, OH, USA. ${ }^{4}$ Department of Brain Tumor and Neuro-Oncology Center, Cleveland Clinic, Cleveland, OH, USA. ${ }^{5}$ Lifespan Physician Group, Rhode Island Hospital, Providence, RI, USA. ${ }^{6}$ Department of Endocrinology, Diabetes and Metabolism, Johns Hopkins University, Baltimore, MD, USA.

Received: 19 May 2020 Accepted: 13 August 2020

Published online: 20 August 2020

\section{References}

1. Rajasoorya C, Holdaway IM, Wrightson P, et al. Determinants of clinical outcome and survival in acromegaly. Clin Endocrinol. 1994;41:95-102.

2. Asa SL. Atlas of Tumor Pathology: Tumors of the Pituitary Gland. Third Series, Fasicle 22. Washington, DC: Armed Forces Institute of Pathology (AFIP); 1998.

3. Jane JA, Starke RM, Elzoghby MA, et al. Endoscopic Transsphenoidal surgery for acromegaly: remission using modern criteria, complications, and predictors of outcome. J Clin Endocrinol Metab. 2011;96:2732-40. 
4. Nachtigall L, Delgado A, Swearingen B, et al. Extensive clinical experience: changing patterns in diagnosis and therapy of acromegaly over two decades. J Clin Endocrinol Metab. 2008;93:2035-41.

5. Mercado M, Gonzalez B, Vargas G, et al. Successful mortality reduction and control of comorbidities in patients with acromegaly followed at a highly specialized multidisciplinary clinic. J Clin Endocrinol Metab. 2014;99:4438-46.

6. Karapanou O, Tzanela M, Christoforaki M, et al. Therapeutic trends and outcome of acromegaly: a single center experience over a 40-year period. Hormones. 2016;15:368-76.

7. Fieffe $S$, Morange I, Petrossians $P$, et al. Diabetes in acromegaly, prevalence, risk factors, and evolution: data from the French acromegaly registry. Eur J Endocrinol. 2011;164:877-84.

8. Mestron A, Webb SM, Astorga R, et al. Epidemiology, clinical characteristics, outcome, morbidity and mortality in acromegaly based on the Spanish acromegaly registry (Registro Espanol de Acromegalia, REA). Eur J Endocrinol. 2004;151:439-46.

9. Reid TJ, Post KD, Bruce JN, et al. Features at diagnosis of 324 patients with acromegaly did not change from 1981 to 2006: acromegaly remains underrecognized and under-diagnosed. Clin Endocrinol. 2010;72:203-8.

10. Evran $M$, Sert M, Tetiker T. Clinical experiences and success rates of acromegaly treatment: the single center results of 62 patients. BMC Endocr Disord. 2014;14:97.

11. Katznelson L, Laws ER, Melmed S, et al. Acromegaly: an Endocrine Society clinical practice guideline. J Clin Endocrinol Metab. 2014;99:3933-51.

12. Cook DM, Ezzat S, Katznelson L, et al. American Association of Clinical Endocrinologists medical guidelines for clinical practice for the diagonosis and management of acromegaly. Endocr Pract. 2004;10:213-25.

13. Park SH, Ku CR, Moon JH, et al. Age- and sex-specific differences as predictors of surgical remission among patients with acromegaly. J Clin Endocrinol Metab. 2018;103:909-16.

14. Maione L, Brue T, Beckers A, et al. Changes in the management and comorbidities of acromegaly over three decades: the French acromegaly registry. Eur J Endocrinol. 2017;176:645-55

15. Arosio M, Reimondo G, Malchiodi E, et al. Predictors of morbidity and mortality in acromegaly:an Italian survey. Eur J Endocrinol. 2012;167(2):189-98.

16. Esposito D, Ragnarsson O, Granfeldt D, et al. Decreasing mortality and changes in treatment patterns in patients with acromegaly from a nationwide study. Eur J Endocrinol. 2018;178:459-69.

17. Gadelha MR, Kasuki L, Lim DST, Fleseriu M. Systemic complications of acromegaly and the impact of the current treatment landscape: an update. Endocr Rev. 2019;40:268-332

18. Geraedts VJ, Andela CD, Stalla GK, et al. Predictors of quality of life in acromegaly: no consensus on biochemical parameters. Front Endocrinol (Lausanne). 2017;8:40.

19. Cuevas-Ramos D, Carmichael JD, Cooper O, et al. A structural and functional acromegaly classification. J Clin Endocrinol Metab. 2015;100:122-31.

20. Bhayana S, Booth GL, Asa SL, et al. The implication of Somatotroph adenoma phenotype to Somatostatin analog responsiveness in acromegaly. J Clin Endocrinol Metab. 2005:90:6290-5.

21. Obari A, Sano T, Ohyama K, et al. Clinicopathological features of growth hormone-producing pituitary adenomas: difference among various types defined by cytokeratin distribution pattern including a transitional form. Endocr Pathol. 2008;19:82-91.

22. Kiseljak-Vassiliades K, Shafi S, Kerr JM, et al. Clinical implications of growth hormone-secreting tumor subtypes. Endocrine. 2012:42:18-28.

23. Fougner SL, Casar-Borota O, Heck A, et al. Adenoma granulation pattern correlates with clinical variables and effect of somatostatin analogue treatment in a large series of patients with acromegaly. Clin Endocrinol. 2012;76:96-102

24. Brzana J, Yedinak CG, Gultekin SH, et al. Growth hormone granulation pattern and somatostatin receptor subtype $2 \mathrm{~A}$ correlate with postoperative somatostatin receptor ligand response in acromegaly: a large single center experience. Pituitary. 2013;16:490-8.

25. Larkin S, Reddy R, Karavitaki N, et al. Granulation pattern, but not GSP or GHR mutation, is associated with clinical characteristics in somatostatin-naïve patients with somatotroph adenomas. Eur J Endocrinol. 2013;168:491-9.

26. Kiseljak-Vassiliades K, Carlson NE, Borges MT, et al. Growth hormone tumor histological subtypes predict response to surgical and medical therapy. Endocrine. 2015:49:231-41.

27. Melmed S, Bronstein MD, Chanson P, et al. A consensus statement on acromegaly therapeutic outcomes. Nat Rev Endocrinol. 2018;14:552-61.
28. Ezzat S, Caspar-Bell GM, Chik CL, et al. Predictive markers for postsurgical medical management of acromegaly: a systematic review and consensus treatment guideline. Endocr Pract. 2019;25:379-93.

29. Yuen KCJ. Medical therapy for acromegaly:towards the practice of a personalised medicine approach. Endocr Pract. 2019;25:396-8.

30. Shanik MH. Limitations of current approaches for the treatment of acromegaly. Endocr Pract. 2016;22:210-9.

\section{Publisher's Note}

Springer Nature remains neutral with regard to jurisdictional claims in published maps and institutional affiliations.
Ready to submit your research? Choose BMC and benefit from:

- fast, convenient online submission

- thorough peer review by experienced researchers in your field

- rapid publication on acceptance

- support for research data, including large and complex data types

- gold Open Access which fosters wider collaboration and increased citations

- maximum visibility for your research: over $100 \mathrm{M}$ website views per year

At BMC, research is always in progress.

Learn more biomedcentral.com/submissions 